\title{
Evaluation of an eddy-permitting finite-element ocean model in the North Atlantic
}

\author{
Sergey Danilov*, Gennady Kivman, Jens Schröter \\ Alfred Wegener Institute for Polar and Marine Research, \\ Postfach 12-01-61, 27515 Bremerhaven, Germany
}

\begin{abstract}
A new version of the 3D finite-element primitive-equation ocean model (FEOM) based on tetrahedron partitioning of the computational domain is applied to simulate the North Atlantic circulation at eddy-permitting resolution $\left(0.2^{\circ}-2^{\circ}\right)$. It relies on a horizontally refined mesh in regions of steep topography and allows the sloping bottom to be represented within the $z$-coordinate vertical discretization, similar to the so called shaved cell approach. It is the first time this approach is used to model large-scale ocean circulation. The FEOM performance in the North Atlantic is compared with that of other models in existence. The meridional overturturning circulation and heat transport compare well to the DYNAMO project models, while the mean sea surface height demonstrates the presence of the Gulf Stream recirculation reproduced only by the ISOPYCNIC model of DYNAMO. The annual mean transports of the Gulf Stream and Deep Western Boundary Current at $27^{\circ} \mathrm{N}$ are of $37 \mathrm{~Sv}$ and $17 \mathrm{~Sv}$ with core velocities of about $1 \mathrm{~m} / \mathrm{s}$ and $12 \mathrm{~cm} / \mathrm{s}$ respectively. Due to flexibiity in mesh refinement the FEOM provides a tool for modelling the influence of small scale phenomena unresolved by current climate models on large scale ocean circulation.
\end{abstract}

Key words: North Atlantic circulation, finite elements, ocean modelling

\footnotetext{
* Corresponding author.

Email addresses: sdanilov@awi-bremerhaven.de (Sergey Danilov), gkivman@awi-bremerhaven.de (Gennady Kivman), jschroeter@awi-bremerhaven.de (Jens Schröter).
}

Preprint submitted to Ocean Modelling 28 November 2003 


\section{Introduction}

It is generally believed that many features of ocean circulation are controlled by bathymetry and are sensitive to the details of coastal lines. Yet the geometry of ocean basins is extremely irregular both horizontally and vertically, and its accurate representation still presents a challenge for most ocean general circulation models (OGCMs). An obvious idea of using terrain-following coordinates faces a problem of pressure gradient errors in hydrostatic approximation, which explains co-existence of two main varieties of OGCMs: $z$ coordinate, sacrificing the accurate representation of bathymetry in favour of pressure gradients and $\sigma$ coordinate, resolving the bathymetry, but prone to pressure gradient errors. Difficulties of $z$ - and $\sigma$-coordinate models are well known and thoroughly discussed in the literature. The partial cell approach suggests improvement over the standard step-wise bathymetry of $z$-coordinate (Adcroft et al., 1997; Pacanowski and Gnanadesikan, 1998). Yet the most rigorous way of accounting for sloping bathymetry in $z$-coordinate models is the concept of shaved or cut cells (Adcroft et al., 1997) that has been applied for years within finite-volume community. Shaved cells cannot however be utilized in the frame of purely finite-difference method on which the most OGCM's rely.

Though partial and shaved cells seem to be quite promising, there is only limited experience of using partial cells (see, i. e., Myers and Deacu, 2004) while no results of using shaved cells in real ocean simulation exist to our knowledge.

The sensitivity of OGCMs to the vertical discretization became a central issue in the late 1990s and was studied thoroughly in several intercomparison projects such as DYNAMO (Willebrand et al., 2001) and DAMÉE-NAB (Chassignet et al., 2000). A much less studied issue is the sensitivity of OGCMs to the discretization of the coast line. Adcroft and Marshall (1998) and Dupont et al. (2003) demonstrated that step-like representation of horizontal boundaries introduced extra viscosity that resulted in a significant bias in the circulation. Probably a more important conclusion of the latter study is that the bias does not diminish with refining the horizontal resolution unless a vorticitydivergence formulation is used and advection is treated in an enstrophyconserving way.

Unstructured grids provide a remedy for resolving these problems, adding also the flexibility of mesh refinement in areas of interest. In a previous paper (Danilov et al., 2004; hereafter referred to as DKS04) we have presented a three-dimensional finite-element ocean model (FEOM) based on the primitive equations and designed for climatic studies. The model uses a 3D tetrahedral mesh based on a 2D unstructured surface triangular mesh, and stratified in the 
vertical direction. It combines terrain-following vertical levels in deep regions with $z$ coordinate in upper $200 \mathrm{~m}$. We tested the model performance on the North Atlantic mesh covering the basin from $7^{\circ} \mathrm{N}$ to $80^{\circ} \mathrm{N}$ with mean resolution of $0.8^{\circ}$, and showed the model to be capable of reproducing the mean heat transport and meridional overturning with realism typical for coarse resolution models.

This paper continues DKS04 and presents an eddy-permitting version of the FEOM, which differs from the coarse-resolution version in two important aspects. First, the coarse resolution version of FEOM for the North Atlantic was not free of pressure gradient errors as it was employing the terrain-following vertical coordinate. Though there are several recipies how to reduce these errors they cannot be supressed totally. However, the 3D tetrahedral FEs provide an opportunity to resolve the sloping bathymetry within the $z$-coordinate framework (no pressure gradient errors) by refining the horizontal resolution locally in regions of steep bathymetry. This approach is similar to the shaved cells used with finite volume discretization and is employed in the new model setup. Second, the new version separates equations for barotropic mode and solves them for barotropic velocities and sea surface height. This provides a considerable gain in performance.

In designing the eddy permitting version, we took into account that the eddy activity is not homogeneous in the ocean. For the North Atlantic it is pronounced in areas of the Gulf Stream, the North Atlantic Current, the Gulf of Mexico, the Caribbean and North Sea which occupy about 1/4 of the total area. Accordingly, we use finer mesh in these areas while the rest is discretized with coarse resolution. Though models based on unstructured grids are more costly in the sense of CPU time per node the use of spatially varying resolution can compensate for this deficiency.

The goal of this paper is two-fold. First, it describes further steps in model development in comparison with the initial setup of DKS04 and presents results of testing the FEOM in the eddy-permitting mode and, second, it compares results with other North Atlantic models (in particular, with those participated in the DYNAMO project) to establish potentiality of using unstructured grids in climate modelling which was questioned (Grifiies et al., 2000).

The paper is organised as follows. We begin with describing the model configuration and highlighting some new (with respect to DKS04) features in Section 2. Section 3 contains analysis of a 10-years simulation run and comparison with a set of other North Atlantic models. Summary and conclusions are presented in Section 4. 


\section{Model design}

\subsection{New features of the FEOM}

The basic principles of the model used in this study were described in DKS04. We mention here two new features which were adopted in the new version.

The coarse-resolution FEOM solved the full set of the 3D primitive equations without splitting them onto barotropic and baroclinic modes. This is computatinally inefficient. However splitting the barotropic and baroclinic dynamics is far from being trivial in the 3D FE context if one tries to ensure that the discretized barotropic equations are a consequence of discretized 3D equations (see DKS04 for details). The main difficulty is caused by the horizontal viscosity operator that cannot be written in terms of the barotropic transport (or velocity).

With the previous coarse resolution version of the model the computational cost of solving 3D unsplit equations was affordable. On the eddy-resolving mesh, the dimension of the problem increased 2.5-fold, and the solver convergence was broken due to a particular character of the problem's stiffness matrix (see DKS04). To essentially decrease the size of the problem, we separated the barotropic subproblem out of the full problem by writting the horizontal viscosity operator as acting on the barotropic velocity in the barotropic subproblem. As long as the viscosity is small the inconsistency between the barotropic and full problems introduced by this approximation is only minor. The sea surface height ( $\mathrm{SSH}$ ) obtained by solving the barotropic subproblem is substituted in the 3D equations which are subsequently solved for the full horizontal velocity. The velocity correction procedure is applied to the horizontal velocity at every time step to eliminate divergence introduced by using stabilization and inconsistent horizontal viscosity.

Another new feature of the model is a mixing parameterization that was absent in the previous version. We utilize a scheme of Gent (1991) that computes the vertical mixing coefficients (for tracers and momentum) as a function of the Richardson number. The horizontal viscosity uses the Smagorinsky formula as in DKS04, but with smaller lower and upper treshold values of $25 \mathrm{~m}^{2} / \mathrm{s}$ and $10^{3} \mathrm{~m}^{2} / \mathrm{s}$ respectively. Our experience is that the Smagorinsky viscosity is actually applied to only a very small portion of the ocean while most of it is stabilized with the treshold viscosity of $25 \mathrm{~m}^{2} / \mathrm{s}$. 
As in DKS04, we use an unstructered triangular surface mesh. A novel strategy of producing the surface mesh was adopted. Given the maximum number of layers and appropriate topography one first computes the number of layers as a function of horizontal coordinates. Next, one tries to arrange the nodes in accordance with the gradient of that function such that the difference in the number of layers between two neighbouring triangles does not exceed one. This approach ensures that the bottom is represented as a continuous piecewise linear surface without any vertical segment. Since the affordable number of $2 \mathrm{D}$ nodes is always limited, the procedure also imposes limitation on the unsmoothness of the bathymetry that could be resolved. In addition to sloping bottom regions described above, we increase the nodal density in coastal and dynamically important areas. Each surface triangle defines a prism which is partitioned by geopotential levels into smaller prisms and finally into tetrahedra.

Fig. 1 depicts the spatially varying horizontal resolution of the model defined as a mean over neighbouring triangles that is much finer (up to about $1 / 5^{\circ}$ ) along the continental slope than in the interior (the model uses approximately 16000 surface nodes and 220000 3D nodes). The vertical discretization consists of 23 layers. The sloping bottom is represented in the way analogous to shaved cells and vertical walls are fully avoided. This flexibility is the main advantage of tetrahedral FE discretization over both $\sigma$ - and $z$-coordinate finite difference models.

\subsection{Boundaries conditions and forcing}

Open boundaries were considered as closed for the velocities. For the temperature and salinity, we introduced sponge zones with relaxation to seasonal climatology. The relaxation time is 5 days at the open boundary and decreases linearly with the distance from the open boundary. The southern buffer zone occupies $5^{\circ}$ while the north-eastern one extends up to $60^{\circ} \mathrm{N}$. This was done to 'parameterize' overflow processes in the Denmark Strait still unresolved at the present mesh. It turns out that otherwise too warm and salty water brought northward by the North Atlantic Current penetrates into the Labrador Sea and makes it too warm and salty after 1 year of simulation.

At the surface, the model was forced by the NCEP wind stress for 1990-1999. The surface temperature and salinity were relaxed to the seasonal Levitus climatology with the relaxation time of 30 days. 


\subsection{Numerical cost}

An important feature of the FEOM is using the backward Euler time stepping scheme which requires an efficient solver to be incorporated. This is very uncommon for the OGCM's which are almost exclusively based on explicit time stepping (except for the implicit free surface used with some models) and the use of solvers is avoided whenever possible.

Here we would like to reiterate (cf. DKS04) that there are two reasons for using the backward time stepping in FEOM. First, discretizing time derivative with finite elements produces the so-called mass matrix that is composed of $L_{2}$ scalar products of basis functions. As such, any scheme of time stepping needs a solver to invert the mass matrix every time step unless it is lumped and thus is replaced by its diagonal approximation. Although computationally more efficient this approximation results however in reduced accuracy, as we have shown in DKS04 on the example of the advection scheme. FEOM uses consistent mass matrices.

Another reason is that iterative solvers always have finite accuracy so that computing second-order derivatives could lead to a noise, expecially on unstructured grids. For that reason viscosity and diffusivity terms are to be included into the operator part. Then, to enhance stability of the scheme (and make larger time steps possible), the Coriolis term in the momentum equation and advection term in the tracer equation are also included into the operator part. This last step makes the stiffness matrices of the dynamic and tracer problem non-symmetric and in this way renders the choice of the solver much more critical (yet tractable).

Griffies et al. (2000) express some concern about solver performance. The basic points stressed by them are:

- difficulties in finding a good first guess;

- poor convergence;

- bad scalability on parallel computers.

The first two points actually depend on the quality of the preconditioner and are never a problem with iterative solvers used in the FEOM (Frickenhaus, 2003). The third point is indeed of concern and requires careful design of the storage and communication structure (efficient node numbering, node partition between the processors and distribution of stiffness matrices are among the important factors). For the current mesh size the model scales up to 8 CPUs.

The CPU cost of FEOM relies heavily on the efficiency of the solver used. To establish the model we had to investigate the solver performance when 
increasing the model size from the coarse to current resolution. It turns out that the PILUT, PETSC and HYPRE solvers used by us (see Frickenhaus (2003) for references to description and original publications) scale linearly with the size of the tracer and full velocity problems (which implies that the number of iterations remains the same). The number of iterations increases for the barotropic subproblem, yet this would be the case with finite-difference models too.

The total CPU cost of FEOM which is about 10 hours per one model year on IBM pSeries 690 is divided between solving the matrix problems and assembling the RHSs and stiffness matrices, and in the current setup the first part takes less than half of the total time. The second part produces a significant CPU load due to combination of several factors: (i) indirect indexing and need to assemble matrices in sparse format which is expensive; (ii) significant increase in the number of floating point operation due to using stabilization; (iii) using the Smagorinsky prameterization for viscosity which implies updating the dynamical stiffness matrix every time step. Taken together, these factors make the CPU cost of FEOM significantly higher than the cost of finitedifference model with the same number of nodes, and this inefficiency comes mostly from using unstructured grids and not from using the backward Euler time stepping and solver. Yet the effective resolution of FEOM could be much finer in some areas than the resolution of a regular-grid model with the same number of computational nodes. FEOM would approach the performance of regular-grid models when compared in terms of effective resolution.

\section{Results}

The resuls presented below were obtained in a 10 years run initialized with the Levitus climatology. This time span is still insufficient for the initial conditions to lose influence, yet no significant trends in SSH are seen at the final stage of integration. The last three years were used for the analysis.

\subsection{Mean sea surface height and variability}

Fig. 2 depicts the mean sea surface height. The Gulf Stream separates somewhat nothern of Cape Hatteras at about $38^{\circ}$. The model resolution in this region is only about $0.3^{\circ}$ (see Fig. 1) which is typically considered insufficient according to the experience gained with other eddy permitting models of comparable $1 / 3^{\circ}$ resolution (see for example, summary of the DYNAMO Group results in Willebrand et al., 2001; we will refer to the models described there as LEVEL, SIGMA and ISOPYCNIC thereafter). Even models of somewhat 
finer resolutions such as 1/4 $4^{\circ} \mathrm{POP}$ (Maltrud et al., 1998), 15-20 km POM (Ezer and Mellor, 2000), 1/6 $6^{\circ}$ POP (Chao et al., 1996) still locate the separation point further to the north. In the vicinity of Cape Hatteras FEOM produces a strong spurious anticyclonic eddy which is a common feature of these $z$-coordinate models. Only very fine resolution models $\left(1 / 10^{\circ} \mathrm{POP}\right.$ of Smith et al., 2000, and 1/12 ${ }^{\circ}$ MICOM of Paiva et al., 1999) perform better. The exception to this rule is a $3 / 4^{\circ}$ version of ROMS (Haidvogel et al., 2000) which even locates the separation point slightly to the south of Cape Hatteras.

FEOM exhibits deep south-west penetration of the Labrador Sea Water which is absent in LEVEL and ISOPYCNIC but stronger in SIGMA where it is of the strength obtained in Smith et al. (2000). Another feature of the mean $\mathrm{SSH}$ of FEOM is a strong Gulf Stream recirculation seen in our Fig. 2. It is comparable to that produced by ISOPYCNIC. Similar to that model, one branch of the FEOM recirculation turns to the east and inspite of the coarse resolution used in the central North Atlantic forms a front at about $30^{\circ} \mathrm{N}$. This front associated with strong wind stress curl off the west coast of Africa at this latitude is clearly visible in POP (see Smith et al., 2000 for discussion) but is not present in the DYNAMO models. Instead ISOPYCNIC produces the front related to the Azores Current not at $35^{\circ}$ as in Smith et al. (2000) but just inbetween these two fronts. We did not impose relaxation towards climatology in the Bay of Cadiz and thus could not produce the Azores Current which appears to be also absent in LEVEL and SIGMA.

The North Atlantic Current (NAC) of FEOM separates from the Grand Banks at about $50^{\circ} \mathrm{N}$ that is in agreement with SIGMA and ISOPYCNIC. As in SIGMA, a branch of the NAC turns to the northwest and penetrates deep into the Labrador Sea. This feature is seemingly caused by topographic effects and is present in a relatively coarse resolution version of ROMS (Haidvogel et al., 2000). Only very fine resolution $z$-coordinate models of Smith et al. (2000) and Böning et al. (1996) are able to reproduce this northwest branch of the NAC.

The FEOM SSH variability is presented in Fig. 3. In the Gulf Stream region it is mainly concentrated around the separation point where its standard deviation exceeds $20 \mathrm{~cm}$. In the remainder part of the Gulf Stream it is of the order of $3 \mathrm{~cm}$ which is lower than the lowest variability in DYNAMO shown by ISOPYCNIC. However, it is worth to remember that the horizontal resolution of FEOM in this area is only about $0.5^{\circ}$ resulting in weaker variability. Yet it is generally comparable with typical values obtained in the DAMÉE-NAB intercomparison project (Chassignet et al., 2000).

The model exhibits strong SSH variability in the Caribbean and Gulf of Mexico which could be attributed to finer spatial resolution there. The standard deviation reaches $12 \mathrm{~cm}$ in the Caribbean Sea and $20 \mathrm{~cm}$ in the Gulf of Mex- 
ico which is notably higher than in $1 / 3^{\circ} \mathrm{MOM}$ and comparable to a $1 / 9^{\circ}$ version (Oschlies, 2002). However the subtle features of circulation are not reproduced properly as they may be sensitive to the detail of spatial resolution (still rather coarse at about $0.6^{\circ}$ in the Central part of the Gulf of Mexico) and local bathymetry (Chassignet, personal communication, 2003). Problems include the direction of eddy propagation in the Gulf of Mexico. Once eddies

enter the Yucatan Channel they turn to the west in FEOM instead of moving in the northwest direction as regional simulations reveal (Morey et al., 2003).

\subsection{Overturning}

The mean meridional overturning stream function of FEOM is presented in Fig. 4. The maximum transport value of the thermohaline cell is $15.5 \mathrm{~Sv}$ which agrees well with LEVEL but is slightly lower than in ISOPYCNIC and SIGMA. The position of the maximum is located at $36^{\circ} \mathrm{N}$ and $1000 \mathrm{~m}$ depth that is just inbetween those obtained in LEVEL and in Smith et al. (2000). The FEOM also exhibits the spurious upwelling around $35^{\circ} \mathrm{N}$ which is associated with the Gulf Stream separation and is traditional to level coordinate models if the Gent and McWilliams (1990) parameterization of mixing is not used (Böning et al., 1995).

The Denmark Strait overflow of the North Atlantic Deep Water (NADW) is relatively weak compared to all three DYNAMO models. This can be explained by relaxation to Levitus climatology considered to poorly represent the East Greenland Current (Willebrand et al., 2001). For this reason, the DYNAMO project used another data set in the northern buffer zone. However, contrary to ISOPYCNIC and SIGMA, FEOM is able to produce strong sinking in the subpolar gyre of $8 \mathrm{~Sv}$ at about $60^{\circ} \mathrm{N}$ which is in agreement with LEVEL and is also supported by Smith et al. (2000).

Another feature of the overturning circulation present in FEOM is sinking of the NADW from $60^{\circ} \mathrm{N}$ to about $25^{\circ} \mathrm{N}$. This sinking is clearly seen in Smith et al. (2000) and ISOPYCNIC, less visible but still present in SIGMA and is absent in LEVEL. One can also notice a drastic deepening of the NADW between $40^{\circ} \mathrm{N}$ and $30^{\circ} \mathrm{N}$ in FEOM. About the same deepening is also present in Smith et al. (2000) at $35^{\circ} \mathrm{N}$ and in ISOPYCNIC at $30^{\circ} \mathrm{N}$.

\subsection{Meridional heat transport}

Fig. 5 depicts the annual mean (over the last 3 years of integration) meridional heat transport. It agrees well with the estimate of MacDonald (1998), SIGMA and ISOPYCNIC for $24^{\circ} \mathrm{N}$ and $48^{\circ} \mathrm{N}$. The heat transport at $36^{\circ} \mathrm{N}$ is 
underestimated by stays within error bars of MacDonald (1998) and agrees with that of LEVEL. It is worth to be reminded in this respect that we relaxed model surface temperature and salinity to Levitus seasonal climatology while all the models of the DYNAMO project were forced by the surface heat flux computed according to Barnier et al. (1995). The latter is shown (Maltrud et al., 1998) to produce notably higher values of the meridional heat flux and overturning. Another feature of the heat transport obtained is that it is notably higher at $60^{\circ} \mathrm{N}$ than in DYNAMO and agrees well with Smith et al. (2000).

The meridional heat transport becomes anomalously low in the vicinity of the open boundary at the north and south as simplified boundary conditions are realized there. The meridional overturning stream function is also affected by closing the open boundaries, so that these parts of the basin circulation should be excluded from consideration.

\subsection{Western Boundary Current}

A distinct feature of the North Atlantic circulation is a narrow Western Boundary Current associated with the major part of the thermohaline circulation. Its realistic representation is a crucial test for the model performance. Fig. 5 depicts a section of the meridional velocity in the vicinity of the western boundary at $27^{\circ} \mathrm{N}$. Due to still insufficient horizontal resolution at the continental shelf which results in oversmoothed bathymery FEOM does not separate the Florida and Antilles Currents as was the case for SIGMA. However, the northward transport appears to be narrower and stronger. Maximum northward velocities are about $1 \mathrm{~m} / \mathrm{s}$ that is as twice as higher than in SIGMA and corresponds well to LEVEL which produced the strongest Florida Current among the DYNAMO models. The volume northward transport between the coast and $78^{\circ} \mathrm{W}$ is $37 \mathrm{~Sv}$ that again agrees well with LEVEL.

The structure of the Deep Western Boundary Current (DWBC) is grossly similar to that produced by SIGMA. As in this model, the core located at the $2000 \mathrm{~m}$ depth is well attached to the continental slope and the deep southward transport has some signature at the surface. Though FEOM has a coarser local resolution in this area than the DYNAMO models, the DWBC has the same horizontal extension of $1^{\circ}$ though still being too wide compared to observations (Lee et al., 1996). The maximum southward velocity at the core of DWBC is $12 \mathrm{~cm} / \mathrm{s}$ which coincides with LEVEL but is slightly lower than $15 \mathrm{~cm} / \mathrm{s}$ observed (Lee et al., 1996) and obtained in SIGMA. The southward volume transport is $17 \mathrm{~Sv}$ which is also in agreement with the DYNAMO models. 


\section{Summary and conclusions}

The results of a 10-year simulation of the North Atlantic circulation with FEOM show that the model is capable of reproducing the main features of the circulation with a skill comparable to that of regular-grid models of finer resolution. We have shown that its mean SSH, meridional overturning and heat flux agree reasonably well with those of $1 / 3^{\circ}$ resolution models. Capability of 3D tetrahedron finite elements in accounting for sloping bottom topography within the $z$-coordinate vertical discretization results in better representation of some details of the circulation such as penetration of the NAC into the Labrador Sea, southward sinking of the NADW and the higher heat transport in comparison with LEVEL. All these problems of LEVEL are overriden at finer resolution (Smith et al., 2000) but at the expence of much higher CPU cost. Due to the potentiality of local mesh refinement in regions of topographically controlled flows such the Labrador Sea, FEOM is able to represent the bathymetry gradients without oversmoothing that is generally needed in $\sigma$-coordinate models to reduce the pressure gradient errors.

The CPU cost of the model is higher than that of the regular-grid model configured with the same number of nodes, which comes mostly from the unstructured character of the FEOM grid, and to a smaller extent, from using solvers. Yet efficiency of the model should be estimated by its ability to reproduce the ocean circulation in dynamically important areas and in most cases depends on local resolution and topography representation. In terms of efficient resolution, FEOM has an affordable CPU cost.

Due to the freedom in representing both the coast lines and bathymetry provided through the FEOM tetrahedral discretization and due to the possibility of refinement in areas of interest, FEOM could serve as a tool to model regional circulations embedded into a coarser-grid global model and thus to overcome the problem of open boundary conditions. One of the main conclusion drawn from the DYNAMO intercomparison is that the large scale ocean circulation is strongly influenced by some localized processes (in particular, by the overflow at the Greenland-Scotland region) and is rather sensitive to small-scale details of the bathymetry (Willebrand et al., 2001). FEOM provides a tool for explicit modelling and investigation the influence of small scale phenomena which are unresolved by current climate models but well reproduced by regional models (in particluar, the overflow processes in a regional version of FEOM by Harig and Schröter, 2004) onto large scale ocean circulation. These will be our next tasks. 


\section{References}

[1] Adcroft, A., Hill, C., and Marshall, J. (1997) Representation of topography by shaved cells in a height coordinate ocean model. Mon. Weath. Rev. 125, $2293-2315$.

[2] Adcroft, A., and Marshall, J. (1998) How slippery are piecewise-constant coastlines in numerical ocean models. Tellus 50A, 95-108.

[3] Barnier, B., Siefridt, L., and Marchesiello, P. (1995) Thermal forcing of a global ocean circulation model using a three-year climatology of ECMWF analyses. J. Mar. Sys. 6, 363-380.

[4] Böning, C. W., Holland, W.R., Bryan, F.O., Danasoglu, G., and McWilliams, J.C. (1995) An overlooked problem in model simulations of the thermohaline circulation and heat transport in the Atlantic Ocean. J. Climate 8, 515-523.

[5] Böning, C. W., Bryan, F.O., Holland, W.R., and Döscher, R. (1996) Deepwater formation and meridional overturning in a high-resolution model of the North Atlantic. J. Phys. Oceanogr. 26, 1142-1164.

[6] Chao, Y., Gangopadhyay, A., Bryan, F.O., and Holland, W.R. (1996) Modeling the Gulf Stream system: How far from reality? Geophys. Res. Lett. 23, 3155-3158.

[7] Chassignet, E.P., Arango, H., Dietrich, D., Ezer, T., Ghil, M., Haidvogel, D.B., Ma, C.-C., Mehra A., Paiva, A., and Sirkes, Z. (2000) DAMÉE-NAB: the base experiment. Dyn. Atmos. Oceans 32, 155-183.

[8] Danilov, S., Kivman, G., and Schröter, J. (2004) A finite element ocean model: principles and evaluation. Ocean Modelling 6, 125-150.

[9] Dupont, F., Straub, D. N., and Lin, C.A. (2003) Influence of a step-like coastline on the basin scale vorticity budget of mid-latitude gyre models. Tellus 55A, 255-272.

[10] Ezer, T., and Mellor, G.L. (2000) Sensitivity studies with the North Atlantic sigma coordinate Princeton Model. Dyn. Atmos. Oceans 32, 185-208.

[11] Frickenhaus, S. (2003) Parallel Solvers for Irregular Sparse Linear Systems. http://www.awipotsdam.de/InfoCenter/IT/WorkingGroups/SciComp/SSolversUG.html

[12] Gent, P.G., and McWilliams, J.C. (1990) Isopycnal mixing in ocean circulation models. J. Phys. Oceanogr. 20, 150-155.

[13] Gent, P.R. (1991) The heat budget of the TOGA-COARE domain in an ocean model. J. Geophys. Res. 96, 3323-3330.

[14] Griffies, S.M., Böning, Bryan, F.O., Chassignet, E.P., Gerdes, R., Hasumi, H., Hirst, A., Treguier, A.-M., and Webb, D. (2000) Developments in ocean climate modeling. Ocean Modelling 2, 123-192.

[15] Haidvogel, D.B., Arango H.G., Hedstrom, K., Beckmann A., MalanotteRizzoli, P., and Shchepetkin, A.F. (2000) Model evaluation experiments in the North Atlantic Basin: simulations in nonlinear terrain-following coordinates. Dyn Atmos. Oceans 32, 239-281.

[16] Harig, S., and Schröter, J. (2004) Modelling dense water overflow with a 
finite element ocean model. Ocean Modelling (submitted).

[17] Lee, T.N., Johns, W., Zantopp, R., and Fillenbaum, E. (1996) Moored observations of western boundary current variability and thermohaline circulation at 26.5 N in the subtropical North Atlantic. J. Phys. Oceanogr. 26, 962-983.

[18] MacDonlad, A.M. (1998) The global ocean circulation: a hydrographic estimate and regional analysis. Progress in Oceanography 41, 281-382.

[19] Maltrud, M.E., Smith, R.D., Semtner, A.J., and Malone, R.C. (1998) Global eddy-resolving ocean simulation driven by 1985-1995 atmospheric winds. J. Geophys. Res. 103,30825-30853.

[20] Myers, P.G., and Deacu D. (2004) Labrador sea freshwater content in a model with a partial cell topographic representation. Ocean Modelling (in press).

[21] Morey, S.L., Zavala-Hidalgo, and O'Brien, J.J. (2003) Impact of vertical resolution on a numerical model of the Gulf of Mexico. Oceans Extended Abstract, 1234-1237.

[22] Oschlies, A. (2002) Improved representation of upper-ocean dynamics and mixed layer depths in a model of the North Atlantic on switching from eddypermitting to eddy-resolving grid resolution. J. Phys. Ocean. 32, 2277-2298.

[23] Pacanowski, R.C., and Gnanadesikan, A. (1998) Transient response in a $z$-level ocean model that resolves topography with partial cells. Monthly Weather Review 126, 3248-3270.

[24] Paiva, A.M., Hargrove, J.T., Chassignet, E.P., and Bleck, R. (1999) Turbulent behaivior of a fine mesh $\left(1 / 12^{\circ}\right)$ numerical simulation of the North Atlantic. J. Mar. Sys. 21, 307-320.

[25] Smith, R.D., Maltrud, M. E., Bryan, F.O., and Hecht, M.W. (2000) Numerical simulation of the North Atlantic Ocean at $1 / 10^{\circ}$. J. Phys. Ocean. $32,1532-1561$.

[26] Willebrand, J., Barnier, B., Böning, C., Dietrich, C., Killworth, P.D., Le Provost, C., Jia, Y., Molines, J.-M., and New, A.L. (2001) Circulation characteristics in three eddy-permitting models of the North Atlantic. Progr. Ocean. 48, 123-161. 


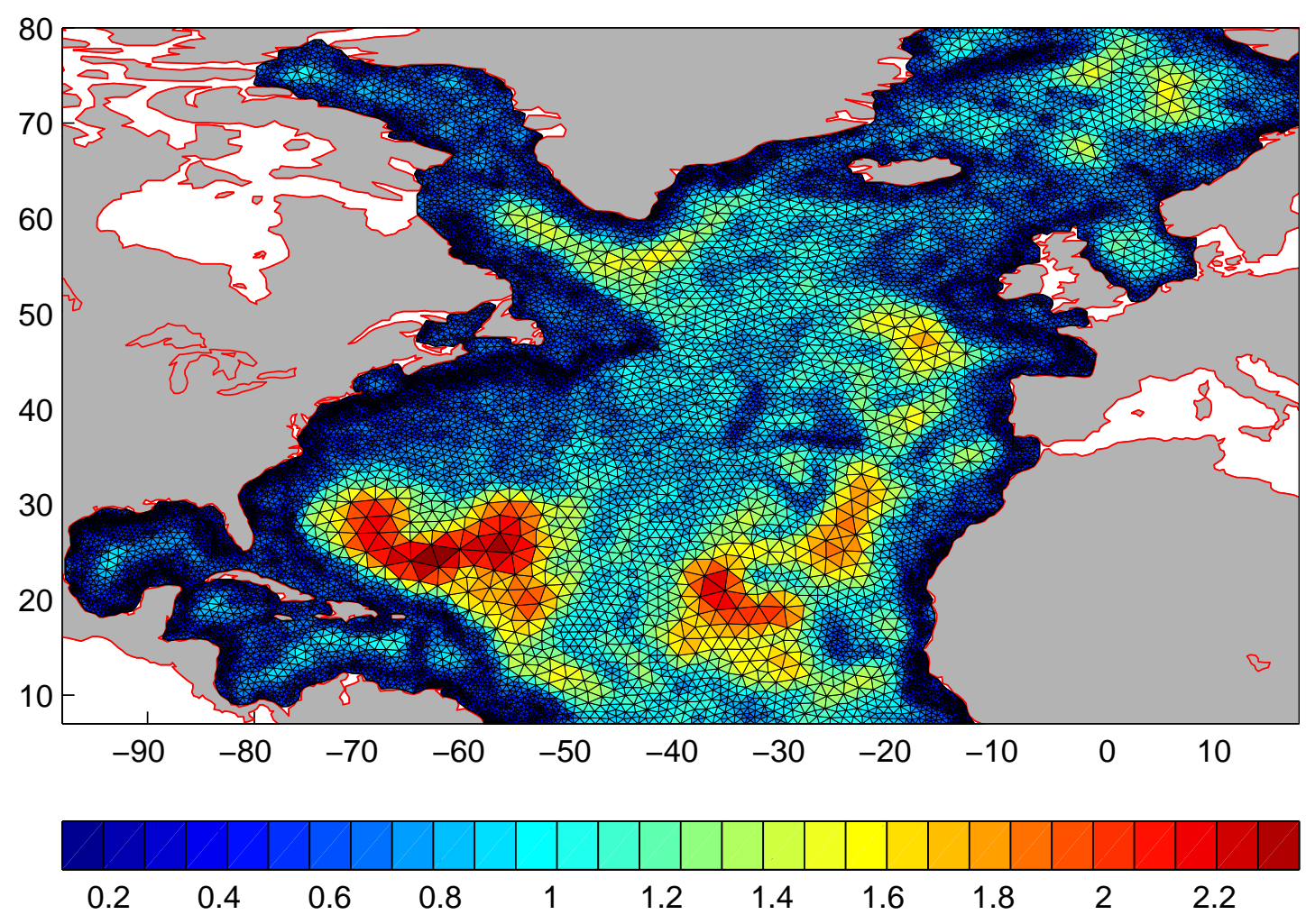

Fig. 1. Resolution in degrees of the North Atlantic mesh averaged over clusters of triangles with a common node. 


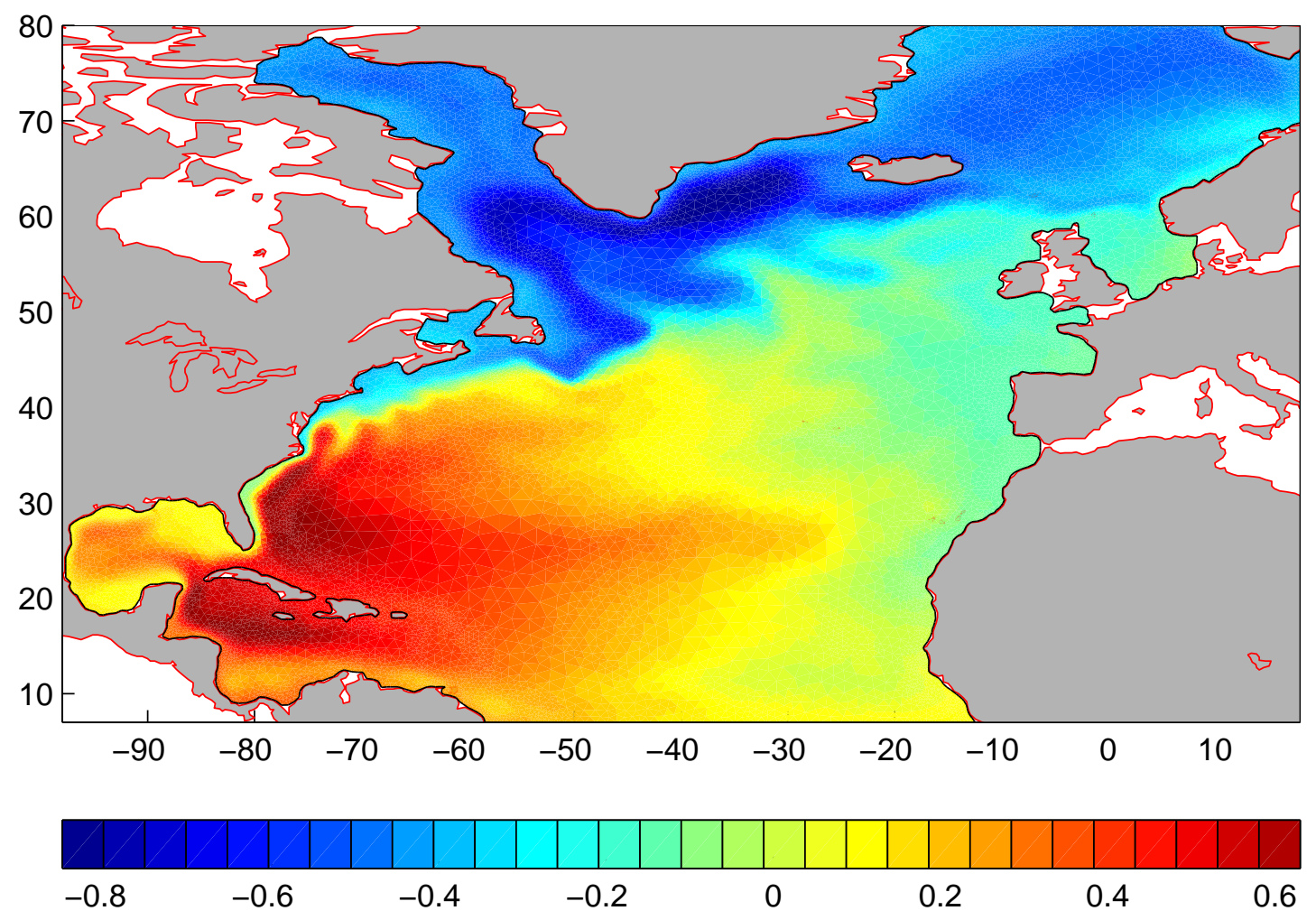

Fig. 2. Mean SSH (m).

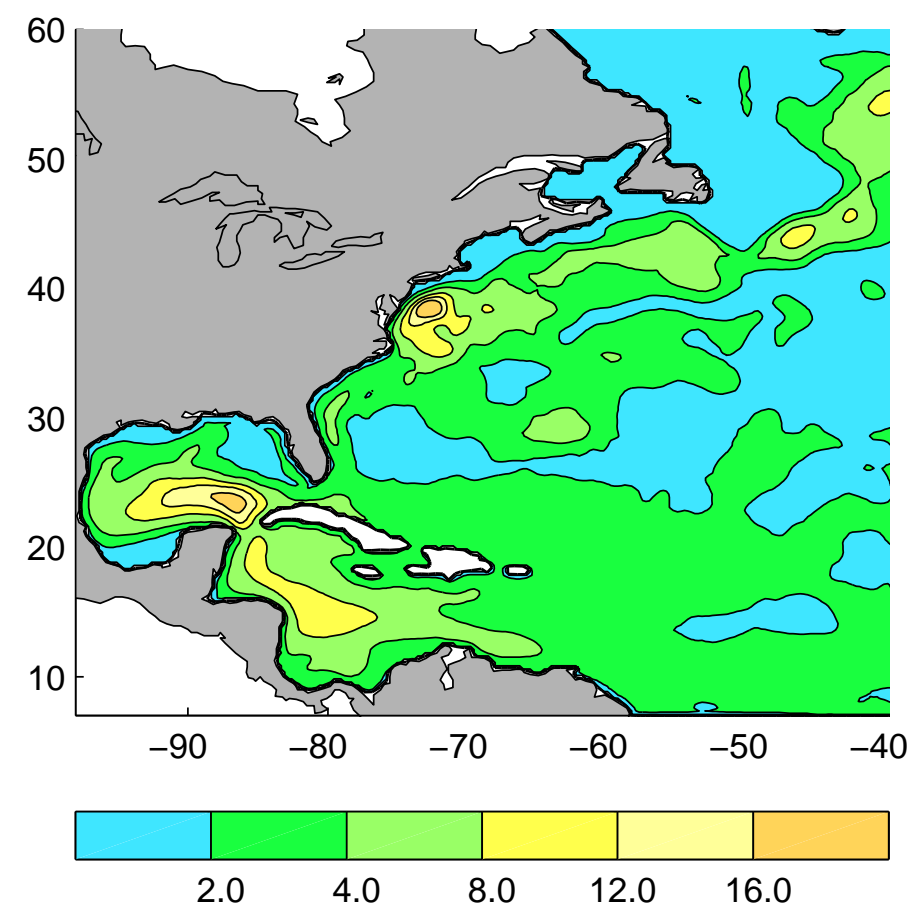

Fig. 3. RMS SSH variability in the Western North Atlantic over the last three years of integration $(\mathrm{cm})$. 

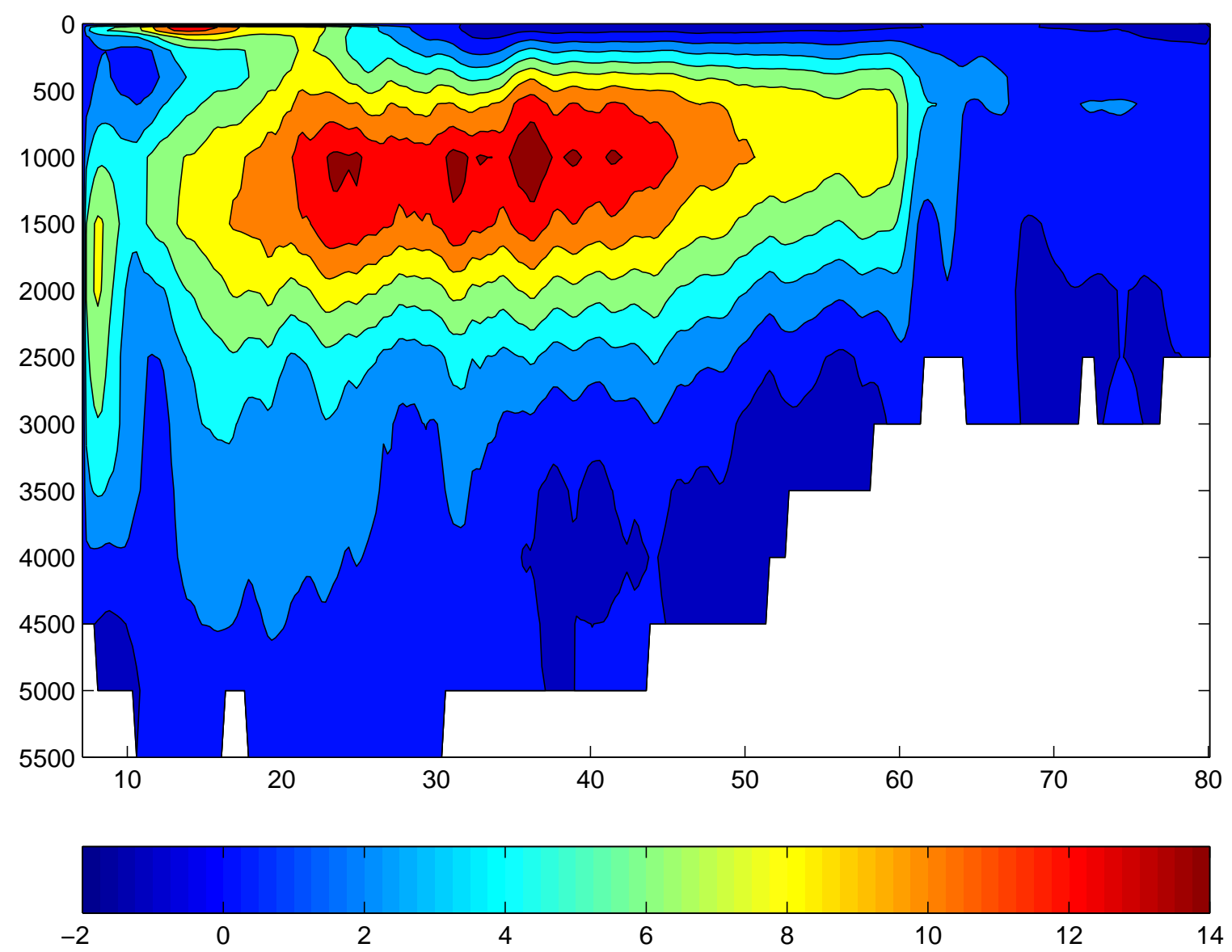

Fig. 4. Meridional overturning stream function $(\mathrm{Sv})$. The maximum value is $15.5 \mathrm{~Sv}$ and is located at $36^{\circ} \mathrm{N}$ and $1000 \mathrm{~m}$ depth. Spurious circulation cells at the south are due to treating the open boundary as closed.

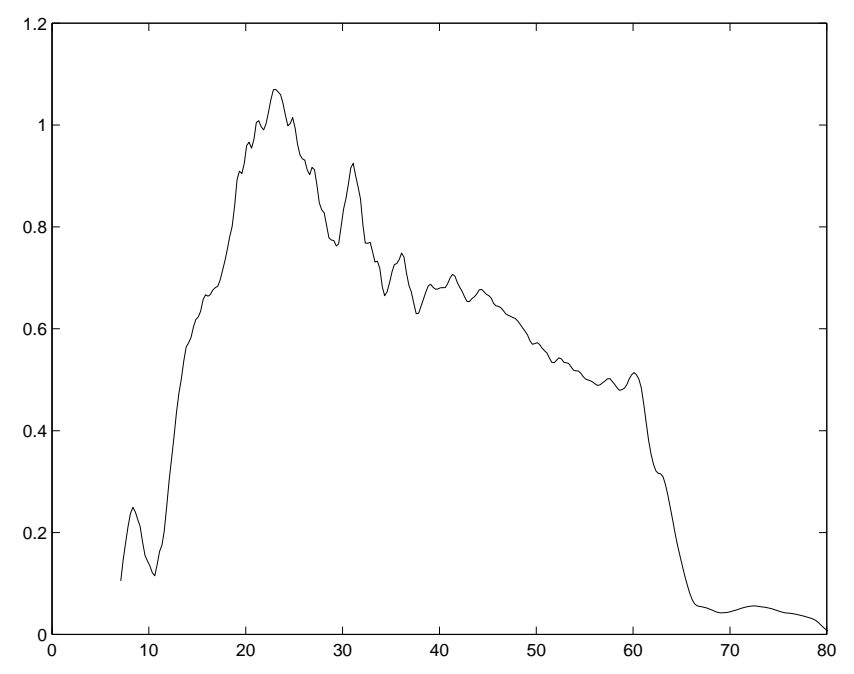

Fig. 5. Meridional heat flux (PW). Anomalous low values of the heat transport at the most southern and northern parts are caused by treating the open boundaries as closed. 


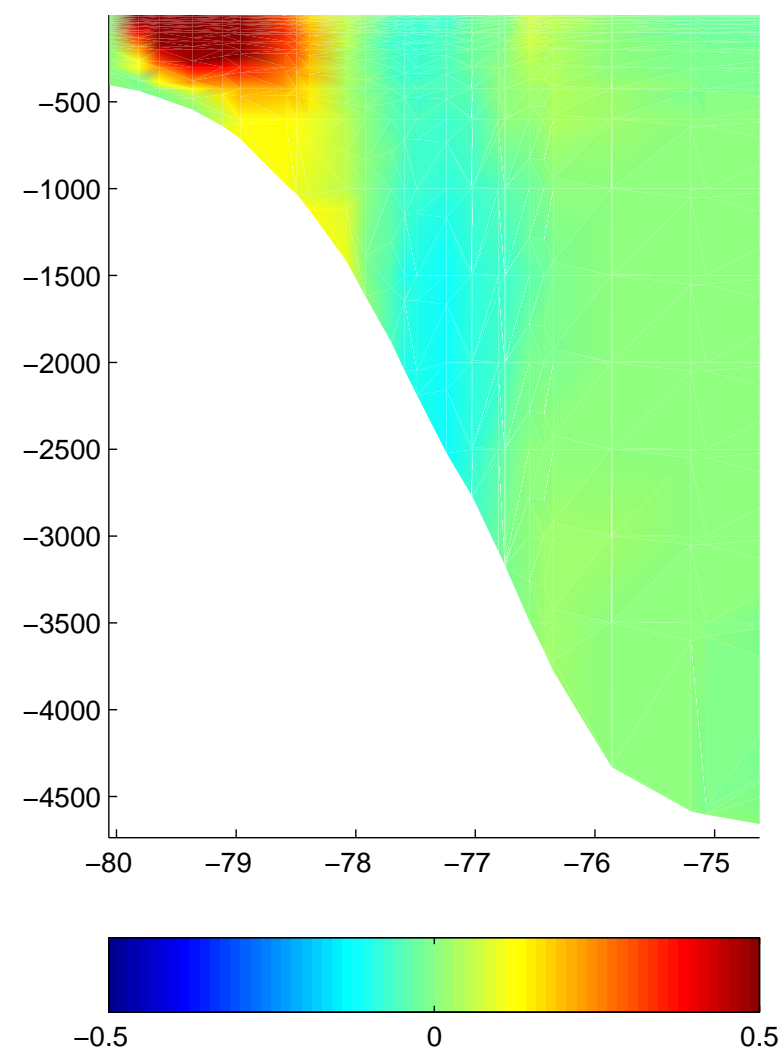

Fig. 6. Meridional velocity cross-section $(\mathrm{cm} / \mathrm{s})$ along $27^{\circ} \mathrm{N}$. The maximum northward and southward velocities are about $1 \mathrm{~m} / \mathrm{s}$ and $12 \mathrm{~cm} / \mathrm{s}$ respectively. The core of the Deep Western Boundary Current is located at the depth of $2000 \mathrm{~m}$. 